\title{
Dynamic and Static Magnetic Resonance Angiography of the Supra-aortic Vessels at 3.0 T
}

\author{
Intraindividual Comparison of Gadobutrol, Gadobenate Dimeglumine, \\ and Gadoterate Meglumine at Equimolar Dose
}

\author{
Jens Harald Kramer, MD, * Elisabeth Arnoldi, MD, * Christopher J. François, MD, $\dagger$ Andrew L. Wentland, MS, $\dagger$ \\ Konstantin Nikolaou, MD, * Bernd J. Wintersperger, MD, ${ }^{*}+$ and Thomas M. Grist, MD $\dagger$
}

\begin{abstract}
Purpose: The purpose of this study was the intraindividual comparison of a $1.0 \mathrm{M}$ and two $0.5 \mathrm{M}$ gadolinium-based contrast agents (GBCA) using equimolar dosing in dynamic and static magnetic resonance angiography (MRA) of the supra-aortic vessels.

Materials and Methods: In this institutional review board-approved study, a total of 20 healthy volunteers (mean \pm SD age, $29 \pm 6$ years) underwent 3 consecutive supra-aortic MRA examinations on a 3.0 T magnetic resonance system. The order of GBCA (Gadobutrol, Gadobenate dimeglumine, and Gadoterate meglumine) was randomized with a minimum interval of 48 hours between the examinations. Before each examination and 45 minutes after each examination, circulatory parameters were recorded. Total GBCA dose per MRA examination was $0.1 \mathrm{mmol} / \mathrm{kg}$ with a $0.03 \mathrm{mmol} / \mathrm{kg}$ and $0.07 \mathrm{mmol} / \mathrm{kg}$ split for dynamic and static MRA, respectively, injected at a rate of $2 \mathrm{~mL} / \mathrm{s}$. Two blinded readers qualitatively assessed static MRA data sets independently using pairwise rankings (superior, inferior, and equal). In addition, quantitative analysis was performed with signal-to-noise ratio (SNR) and contrast-to-noise ratio (CNR) evaluation as well as vessel sharpness analysis of static MRA using an in-house-developed semiautomated tool. Dynamic MRA was evaluated for maximal SNR. Statistical analysis was performed using the Cohen $\kappa$, the Wilcoxon rank sum tests, and mixed effects models.
\end{abstract}

Results: No significant differences of hemodynamic parameters were observed. In static MRA, Gadobutrol was rated superior to Gadoterate meglumine $(P<0.05)$ and equal to Gadobenate dimeglumine $(P=0.06)$ with good to excellent reader agreement $(\kappa, 0.66-0.83)$. In static MRA, SNR was significantly higher using $1.0 \mathrm{M}$ Gadobutrol as compared with either $0.5 \mathrm{M}$ agent $(P<0.05$ and $P<0.05)$ and CNR was significantly higher as compared with Gadoterate meglumine $(P<0.05)$, whereas CNR values of Gadobutrol data sets were not significantly different as compared with Gadobenate dimeglumine $(P=0.13)$. Differences in CNR between Gadobenate dimeglumine and Gadoterate meglumine were not significant $(P=0.78)$. Differences in vessel sharpness between the different GBCAs were also not significant $(P>0.05)$. Maximal SNR in dynamic MRA using Gadobutrol was significantly higher than both comparators at the level of the proximal and distal internal carotid artery $(P<0.05$ and $P<0.05 ; P<0.05$ and $P<0.05)$.

Conclusions: At equimolar doses, $1.0 \mathrm{M}$ Gadobutrol demonstrates higher SNR/CNR than do Gadobenate dimeglumine and Gadoterate meglumine, with superior image quality as compared with Gadoterate meglumine for dynamic and

Received for publication August 16, 2012; and accepted for publication, after revision October 2, 2012.

From the *Institute for Clinical Radiology, Ludwig Maximilians University Hospital Munich, Munich, Germany; †Department of Radiology, The University of Wisconsin-Madison, Madison, WI; and \$Department of Medical Imaging, University of Toronto, Toronto, Canada.

Conflicts of interest and sources of funding: Supported by Bayer Healthcare.

The authors report no conflicts of interest.

Reprints: J. Harald Kramer, MD, Institute for Clinical Radiology, Ludwig Maximilians University Hospital Munich, Marchioninistr. 15, 81377 Munich, Germany. E-mail: harald.kramer@med.lmu.de.

Copyright (C) 2013 by Lippincott Williams \& Wilkins

ISSN: 0020-9996/13/4803-0121 static carotid MRA. Despite the shortened bolus with Gadobutrol, no blurring of vessel edges was observed.

Key Words: magnetic resonance angiography, contrast media, carotid arteries, vessel blurring, image quality, Gadobutrol, Gadobenate dimeglumine, Gadoterate meglumine

(Invest Radiol 2013;48: 121-128)

A cute arterial events are among the most frequent causes of premature death in the developed world, and cerebrovascular events have replaced cardiac events as the leading cause of death. Therefore, interest in imaging of the supra-aortic vessels has significantly increased. ${ }^{1-3}$ In most vascular territories, digital subtraction angiography still remains the standard of reference. ${ }^{4,5}$ However, the known drawbacks of digital subtraction angiography have spurred the development of noninvasive techniques. ${ }^{6,7}$ Because of recent developments, magnetic resonance angiography (MRA) is now routinely used, along with computer tomographic angiography or ultrasound. ${ }^{7-10}$ The image quality of MRA is enhanced with the use of dedicated gadolinium-based contrast agents (GBCA) ${ }^{11-14}$ which improve signal-tonoise ratio $(\mathrm{SNR})$ and contrast-to-noise ratio $(\mathrm{CNR}){ }^{14-16}$

However, because of the description of a potential link between the applications of GBCAs and the development of nephrogenic systemic fibrosis (NSF), ${ }^{17,18}$ efforts are made to reduce the amount of GBCA administered for comprehensive diagnostic imaging of the arterial vasculature. ${ }^{8,19}$ Although earlier studies proposed higher doses of standard GBCA, ${ }^{20-22}$ currently, MRA examinations are typically performed at single doses $(0.1 \mathrm{mmol} / \mathrm{kg})$. Furthermore, the current state-of-the-art MRA of the cervical arteries comprises high-resolution static and dynamic imaging. Previously published studies have shown that, using standard GBCA, excellent image quality is possible using a single dose, and good image quality is possible even with lower than single doses. ${ }^{9,23-25}$ However, reducing the amount of injected contrast agent results in a shortening of the bolus because a certain injection rate is needed to keep the bolus tight. Sampling of the peripheral parts of k-space without the presence of contrast agent bolus theoretically leads to blurring of the vessel edges. ${ }^{26}$

We hypothesized that, in supra-aortic MRA, 1.0 M Gadobutrol is noninferior to $0.5 \mathrm{M}$ Gadobenate dimeglumine and superior to Gadoterate meglumine when applied at single equimolar dose. The purpose of this study therefore was to intraindividually compare all 3 GBCAs in dynamic and static supra-aortic MRA with application of identical imaging and dosage parameters in respect to qualitative image quality and quantitative signal parameters.

\section{MATERIALS AND METHODS}

\section{Study Population}

This single-center, blinded, prospective, intraindividual comparison study was approved by the institutional review board, and written informed consent was obtained from all study volunteers. 
Twenty-two healthy men (mean \pm SD age, $28.7 \pm 5.9$ years; range, 21-41 years) without history of vascular or renal disease, without contraindication against any of the investigational GBCA, or without general magnetic resonance (MR) contraindications were included between June 2011 and August 2011.

Before inclusion in the study, a blood sample was drawn from every volunteer and the glomerular filtration rate was calculated using the Cockroft-Gault formula (eGFR) and confirmed to be greater than $60 \mathrm{~mL} / \mathrm{min}$ per $1.73 \mathrm{~m}^{2}$.

\section{Magnetic Resonance Imaging and Study Protocol}

All MRA examinations were performed on a $3.0 \mathrm{~T}$ 32-channel whole-body open-bore MR system (Magnetom Verio; Siemens Healthcare, Erlangen, Germany). For signal reception, a 12-element head array coil in combination with a 4-element neck surface coil was used.

For static MRA, a standard 3-dimensional fast low-angle shot sequence with parameters optimized for 3.0 T was used. Because image quality in terms of SNR is regarded as sufficient at $1.5 \mathrm{~T}$, the potential increase in signal at 3.0 T was invested in increasing spatial resolution and shortening the acquisition time. Readout was done in a centric fashion with a time to center of 1 second. Because optimizing sequence parameters for 1 agent would penalize both remaining agents, the sequence settings were chosen to reflect the state-of-theart 3.0 T MRA parameters. Detailed sequence parameters can be found in Table 1. For dynamic MRA, a time-resolved angiography with interleaved stochastic trajectories sequence was used. ${ }^{27}$ This sequence is based on a view-sharing technique and facilitates in setting the size of the central region of k-space, which is sampled at every time point, and the sampling density of the remaining peripheral part of k-space. Because, in this study, a high spatial resolution static data set was acquired as well, dynamic MRA was optimized for temporal information. Hence, spatial resolution and potentially available signal were sacrificed to increase temporal resolution. Detailed sequence information can be found in Table 1 .

The volunteers were imaged at 3 different time points with the 3 different contrast agents administered in a randomized fashion and a delay of 48 hours to 30 days between individual GBCA applications. Hemodynamic parameters (eg, blood pressure, heart rate) were recorded before each scan, and an 18-Gauge venous access was placed in the right antecubital vein. Total GBCA dose per examination was $0.1 \mathrm{mmol} / \mathrm{kg}$ body weight (BW) with a $70 \%-30 \%$ split for static and dynamic MRA, respectively. Static MRA was always performed as the first of both examinations to exclude any effect of the previously administered contrast agent on SNR and CNR measurements on static

TABLE 1. Sequence Details of Static MRA and Dynamic MRA

\begin{tabular}{lcc}
\hline & Static MRA & Dynamic MRA \\
\hline Acquisition time, seconds & 21.6 & 98 \\
Temporal resolution, seconds & - & 1.85 ; interp, 0.925 \\
Parallel imaging factor & 4 & 3 \\
Spatial resolution, mm ${ }^{3}$ & $0.8 \times 0.8 \times 0.8$ & $1.4 \times 1.4 \times 1.4$ \\
TR (repetition time), milliseconds] & 3.25 & 2.46 \\
TE (echo time), milliseconds & 1.26 & 0.92 \\
Flip angle, degrees & 21 & 18 \\
Matrix & $576 \times 342$ & $256 \times 176$ \\
FOV, mm & (26) \\
Bandwidth, Hz & $450 \times 267$ & $350 \times 240$ \\
\hline
\end{tabular}

Note the excellent spatial resolution of both techniques and the temporal resolution of less than 2 seconds per frame of dynamic MRA interpolated to 0.925 seconds per frame.
TABLE 2. Calculation of Combined Results From Blinded Image Quality Reading

\begin{tabular}{lcc}
\hline Reader 1 & Reader 2 & Combined Result \\
\hline CA A is superior & CA A is superior & CA A is superior \\
CA A is superior & both equal & CA A is superior \\
CA A is superior & CA B is superior & both equal \\
both equal & CA A is superior & CA A is superior \\
both equal & both equal & both equal \\
both equal & CA B is superior & CA B is superior \\
CA B is superior & CA A is superior & both equal \\
CA B is superior & both equal & CA B is superior \\
CA B is superior & CA B is superior & CA B is superior \\
\hline
\end{tabular}

CA A indicates contrast agent $\mathrm{A}$; CA B, contrast agent $\mathrm{B}$.

MRA data sets. All GBCA injections were performed using a power injector (Spectris Solaris; Medrad). For the evaluation of bolus arrival time, a test bolus technique with a fixed dose of $1 \mathrm{~mL}$ of contrast agent injected at a flow rate of $2 \mathrm{~mL} / \mathrm{s}$, followed by a $20 \mathrm{~m} / \mathrm{L}$ saline chase injected at the same rate, was used. Both static and dynamic MRA were performed with the same injection parameters. ${ }^{9,27}$ After each examination, hemodynamic parameters were reassessed and the volunteers were monitored for the occurrence of adverse events for 60 minutes.

\section{Contrast Agents}

The following Gd-based contrast agents (GBCAs) were used for this study: Gadobutrol (Gadovist/Gadavist; Bayer Healthcare, Berlin, Germany), Gadobenate dimeglumine (Multihance; Bracco Diagnostics, Milan, Italy), and Gadoterate meglumine (Dotarem; Guerbet, Roissy CdG Cedex, France).

Gadobutrol is a $1.0 \mathrm{M}$ macrocyclic GBCA with a relaxivity $\left(\mathrm{L} \mathrm{mmol}^{-1} \mathrm{~s}^{-1}\right)$ of $5.0(4.7-5.3)$ at 3.0 T. Gadobenate meglumine is a $0.5 \mathrm{M}$ linear GBCA with a weak transient protein binding and a relaxivity of $5.5(5.2-5.8)$ at $3.0 \mathrm{~T}$. Gadoterate meglumine is a $0.5 \mathrm{M}$ macrocyclic GBCA and a relaxivity of $3.5(3.3-3.7)$ at $3.0 \mathrm{~T}^{14}$

\section{Image Quality Evaluation}

Qualitative image quality of all static MRA data sets was assessed independently by 2 radiologists with more than 10 years of experience in MRA using pairwise comparisons. The readers were asked to give their overall impression on whether a data set was better, equal, or worse than the given comparator. The readers included measures such as vessel conspicuity and vessel homogeneity in their judgment but excluded factors that are not related to a certain contrast agent, for example, venous enhancement. Both observers were blinded to any information about the used contrast agent, and after the independent evaluation, assessments of both readers were combined, as shown in Table 2.

Quantitative analysis of both static and dynamic MRA data sets was performed on the basis of the assessment of SNR and CNR at the level of the proximal internal carotid artery (ICA) next to the carotid bifurcation and at the distal ICA just proximal to the carotid T at the level of the skull base. Because the static and dynamic MRA acquisitions used parallel imaging techniques, SNR and CNR evaluations were performed by applying the difference method, as previously described by Dietrich et al. ${ }^{28}$ Hence, 2 consecutive unenhanced data sets were acquired before the contrast agent application and subtracted from each other. The SD within a region of interest (ROI) positioned in the subtracted data set at identical position as the signal measurement in the enhanced data set was defined as image noise for that specific location. For calculation of 
contrast between the vessel signal and the surrounding tissues, an ROI was positioned within the masseter muscle.

In addition to SNR and CNR evaluation, a semiautomated quantitative evaluation of the vessel edge sharpness was performed using an in-house-developed MATLAB-based tool (MathWorks, Natick, MA). After a user-based identification of the center of the vessel of interest on an axial reformatted slice, the tool automatically generated 6 equally spaced radial spokes in 30-degree intervals. Each of the 6 spokes provided a line profile and 2 vessel edges, for a total of 12 vessel edges. Vessel edge sharpness $(\mathrm{mm})$ was defined as the distance between the $20 \%$ and $80 \%$ of the maximum signal intensity on each side of the line profile (Fig. 1). Vessel edge sharpness was averaged over all 12 vessel edges.

\section{Statistical Analysis}

For the assessment of contrast agent superiority via qualitative pairwise preference comparisons, a Wilcoxon signed rank test and the Cohen $\kappa$ for the assessment of interreader agreement were used. For the assessment of quantitative parameters such as SNR, CNR, and vessel edge sharpness, linear mixed effects models, Kruskal-Wallis tests, and the Wilcoxon rank sum test were used. $P<$ 0.05 (2-sided) was considered as statistically significant. Computations were done using measurements of all the volunteers; given values are mean values. Given SDs are mean values of measured SDs. All computations were done in R for Windows, version 2.12.1 (R Development Core Team, 2009).

\section{RESULTS}

All contrast agents were administered at $0.1 \mathrm{mmol} / \mathrm{kg}$ with a $70 \%-30 \%$ split for static and dynamic MRA. This resulted in a mean $\pm \mathrm{SD}$ volume of $11.2(1.1) \mathrm{mL}$ for the $0.5 \mathrm{M}$ agents, $5.6(0.6) \mathrm{mL}$ for gadobutrol for static MRA, and $4.8(0.5) \mathrm{mL}$ and $2.4(0.2) \mathrm{mL}$ for the $0.5 \mathrm{M}$ and 1.0 $\mathrm{M}$ agents for dynamic MRA, respectively.

\section{Qualitative Analysis}

In none of the volunteers circulatory parameters in terms of blood pressure and heart rate differed significantly from each other between the three different exams. No significant differences were observed between the pre-examination and postexamination measurements. In addition, because the investigated cohort consists of healthy, relatively young men, no significantly different circulatory parameters occurred interindividually.

Combined results of the pairwise comparison of static MRA data sets acquired with Gadobutrol, Gadobenate dimeglumine, and Gadoterate meglumine showed Gadobutrol superior to Gadobenate dimeglumine in $10(50 \%)$ cases and to Gadoterate meglumine in 17 $(85 \%)$ cases. It was rated as equal to the comparator in 7 and 2 cases (35\% and 10\%) when compared with Gadobenate dimeglumine and Gadoterate meglumine, respectively. Gadobutrol was judged as inferior compared with Gadobenate dimeglumine and Gadoterate meglumine in 3 and 1 case $(15 \%$ and $5 \%)$, respectively. Gadobenate dimeglumine was judged as superior, equal, or inferior as compared with Gadoterate meglumine in 10,5 , and 5 cases $(50 \%, 25 \%$, and $25 \%$; Fig. 2; Table 3). The Wilcoxon rank test revealed Gadobutrol to be insignificantly different compared with Gadobenate dimeglumine $(P=0.057)$ but significantly superior as compared with Gadoterate meglumine $(P<0.005)$. Gadobenate dimeglumine was rated as not significantly different from Gadoterate meglumine $(P=0.21)$. The interreader agreement for this qualitative pairwise evaluation was good to excellent with Cohen $\kappa$ values of 0.66 to 0.83 for the comparison of Gadobutrol with Gadobenate dimeglumine $(\mathrm{k}=0.66)$ and Gadoterate meglumine $(\mathrm{k}=0.83)$ as well as Gadobenate dimeglumine and Gadoterate meglumine $(\mathrm{k}=0.66)$ (Table 4$)$.

\section{Quantitative Analysis}

\section{Static MRA}

Gadobutrol featured significantly higher SNR at the level of the proximal ICA as compared with Gadobenate dimeglumine $(P<0.05)$ and Gadoterate meglumine $(P<0.05)$, with values of

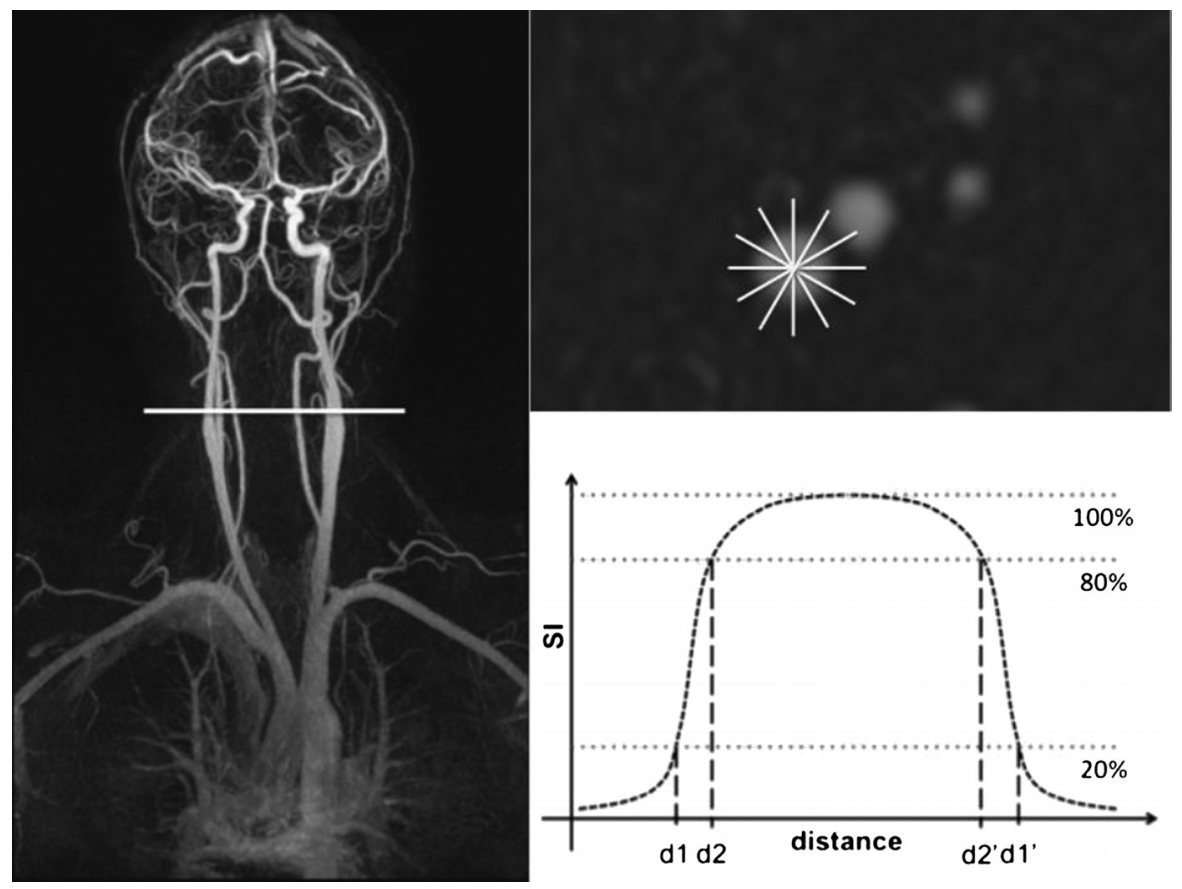

FIGURE 1. Example of static MRA of the carotid arteries showing the level of the vessel sharpness evaluation and the reconstructed axial slice including vessel profile lines. The graph shows an exemplary vessel signal profile with $\mathrm{d} 1$ and $\mathrm{d} 1{ }^{\prime}$ representing $20 \%$ of the maximal signal, $\mathrm{d} 2$ and $\mathrm{d} 22^{\prime}$ representing $80 \%$ of the maximal signal and full width half max level. 

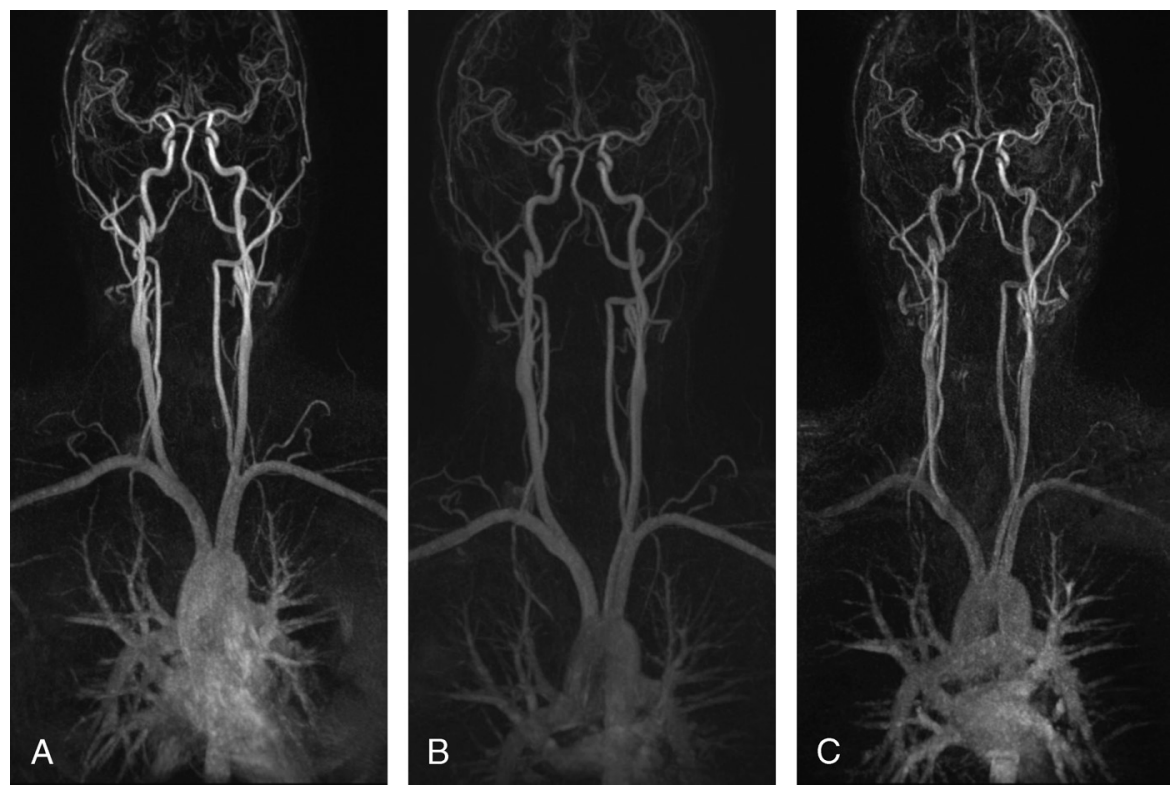

FIGURE 2. Examples of the static MRA examinations acquired with Gadobutrol (A), Gadobenate dimeglumine (B), and Gadoterate meglumine $(C)$. Note the already visually assessable differences in signal intensity and image contrast. No significant differences in edge blurring are visible.

$87.4 \pm 4.2,47.8 \pm 4.0$, and $44.9 \pm 3.5(55 \%$ and $51 \%$ of SNR achieved with gadobutrol), respectively. In the distal ICA at the level of the skull base, SNR was not significantly different between all agents with values of $81.7 \pm 5.6,58.4 \pm 7.1(71 \%)$, and $52.0 \pm 5.8$ (64\%) for Gadobutrol, Gadobenate dimeglumine, and Gadoterate meglumine, respectively. Contrast-to-noise ratio in MRA data sets acquired with Gadobutrol again was superior as compared with Gadobenate dimeglumine $(P=0.13)$ and significantly better than Gadoterate meglumine $(P=0.03)$, with values of $71.1 \pm 3.8,36.9 \pm 3.9$ $(52 \%)$, and $30.5 \pm 3.5(43 \%)$, respectively (Fig. 3). Calculation of vessel sharpness revealed no significant different results for all 3 agents with values of $1.17 \pm 0.24 \mathrm{~mm}, 1.05 \pm 0.18 \mathrm{~mm}$, and $1.11 \pm$ $0.27 \mathrm{~mm}$ for Gadobutrol, Gadobenate dimeglumine, and Gadoterate meglumine, respectively.

\section{Dynamic MRA}

The absence of significant interindividual and intraindividual differences in terms of hemodynamic parameters is reflected by the

TABLE 3. Results of Combined Qualitative Image Quality

Assessment

\begin{tabular}{lcr}
\hline & Frequency & \% \\
\hline Gadobutrol $>$ Gd-BOPTA & 10 & 50 \\
Gadobutrol $<$ Gd-BOPTA & 3 & 15 \\
Gadobutrol = Gd-BOPTA & 7 & 35 \\
Gadobutrol $>$ Gd-DOTA & 17 & 85 \\
Gadobutrol $<$ Gd-DOTA & 1 & 5 \\
Gadobutrol = Gd-DOTA & 2 & 10 \\
Gd-BOPTA $>$ Gd-DOTA & & \\
Gd-BOPTA $<$ Gd-DOTA & 10 & 50 \\
Gd-BOPTA $=$ Gd-DOTA & 5 & 25 \\
\hline
\end{tabular}

Gd-BOPTA indicates gadobenate dimeglumine; Gd-DOTA, gadoterate meglumine. finding that the mean maximal SNR is reached within a range of 3 time frames (18.5-21 sec. p.i. in the proximal ICA and 20-22 sec. p.i. at the level of the skull base) in all examinations. Dynamic MRA such as static MRA showed higher SNR of Gadobutrol as compared with both comparators at the 2 investigated levels in the proximal ICA and at the level of the skull base (all $P<0.05$ ). At the level of the proximal ICA, Gadobutrol, Gadobenate dimeglumine, and Gadoterate meglumine reached a maximal SNR of $76.73,59.41(77 \%)$, and $43.99(57 \%)$, respectively. At the level of the skull base, agents reached a maximal SNR of $117.77,83.77(71 \%)$, and $71.22(60 \%)$, respectively (Fig. 4).

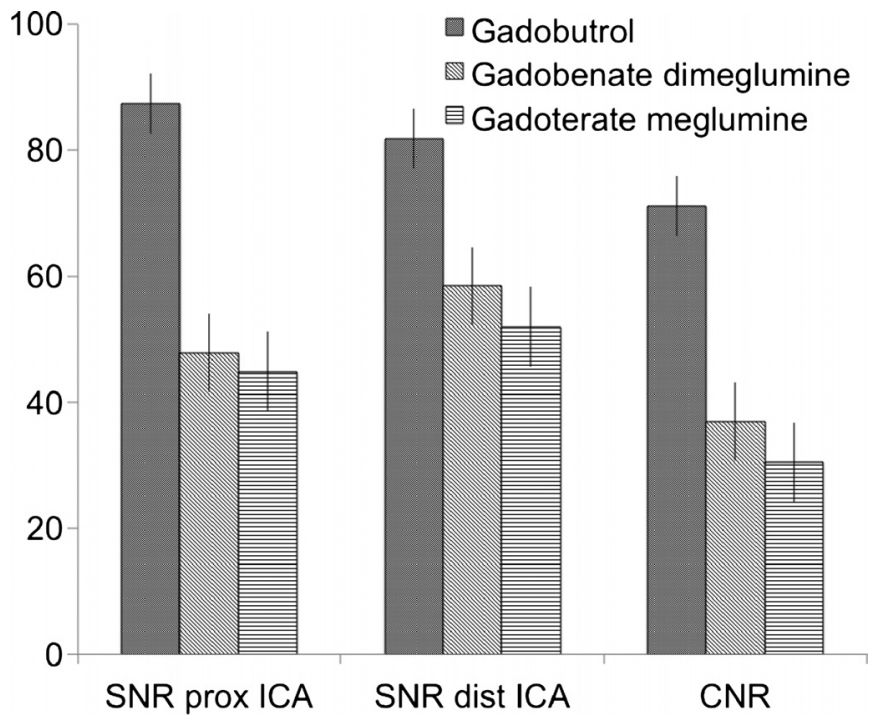

FIGURE 3. Signal-to-noise ratio at both levels of interest and CNR for gadobutrol, Gadobenate dimeglumine, and Gadoterate meglumine. 

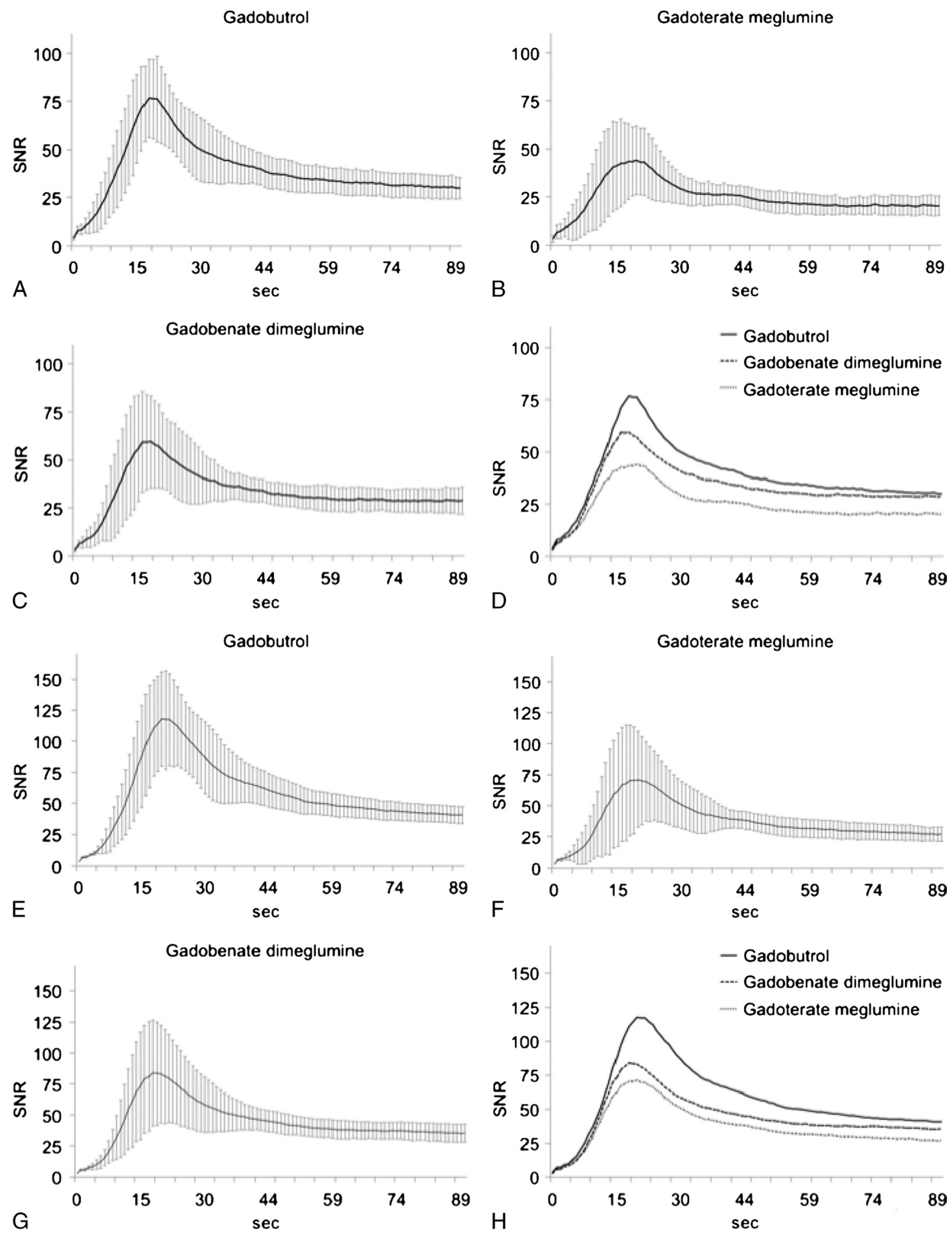

FIGURE 4. Signal-time curves for dynamic MRA acquired with the 3 different GBCA at the level of the proximal ICA (A-C) and at the level of the skull base $(E-G)$. D and $\mathrm{H}$ show signal-time curves for all GBCA in comparison at respective locations. Note that the SNR curve of Gadobenate dimeglumine, although lower at the first-pass peak and the initial curve decline, approaches the SNR of Gadobutrol in the very last stages of the dynamic MRA data acquisition.

\section{DISCUSSION}

Recent publications have shown that MRA has evolved as a method for accurate imaging of the head and neck vasculature at high image quality over the last decade. ${ }^{7,9,29,30}$ However, there are several factors influencing image quality of such examinations. High field strength 3.0 T MR systems, besides 1.5 T MR systems, nowadays can be regarded as the clinical standard, which is beneficial for multiple MRI applications, particularly MRA. ${ }^{31-34}$ Although it is 


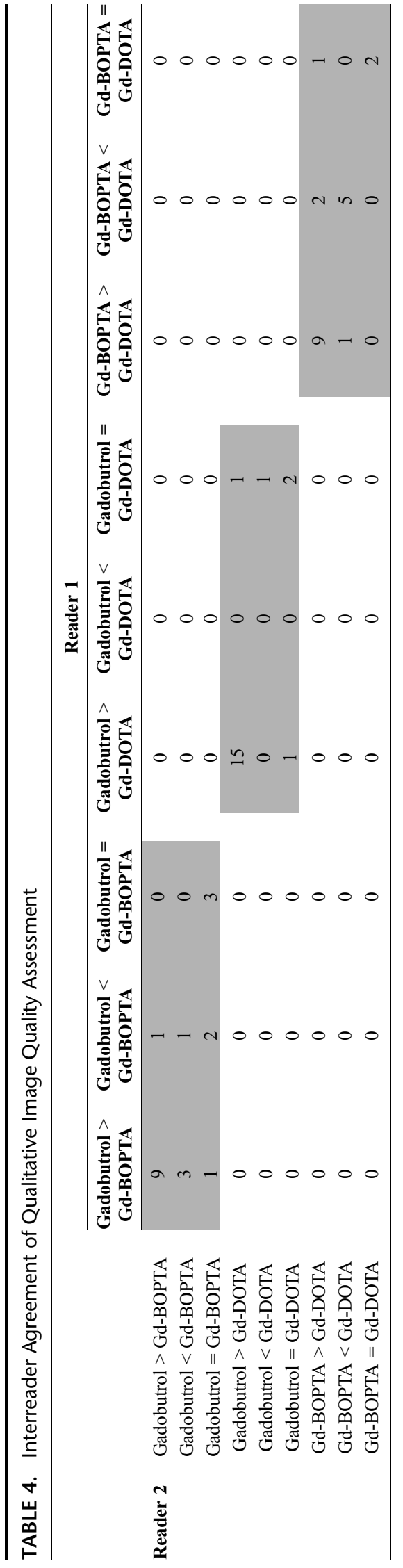

possible to do MRA with many different GBCA, the properties of these agents differ and may affect the image quality of MRA. These characteristics are influenced by multiple different factors such as molecular structure and size, interaction with blood components, and concentration, whereas all of them influence the relaxivity of an agent. ${ }^{14}$ In this study, 3 main groups of available GBCA were compared: (a) agents without interaction with blood components at standard concentration (Gadoterate meglumine), (b) agents with interaction with blood components at standard concentration (Gadobenate dimeglumine), and $(c)$ agents without interaction with blood components at higher concentration (Gadobutrol). Agents featuring interaction with blood components at a concentration higher than $0.5 \mathrm{M}$ are not currently clinically available. Previous publications have already shown that interaction with or binding to certain blood components, in addition to higher Gd concentration, are beneficial as compared with the standard GBCA for certain MRI applications, for example, imaging of brain lesions. ${ }^{12,35-38}$ However, because some agents change their characteristics between injection, imaging, and excretion, results from morphological imaging studies are not totally transferable to MRA. In addition, results from MRA studies of certain anatomical areas are not transferrable to other areas without careful interrogation because injection volume and injection rate, in addition to contrast agent travel time, influence the concentration of contrast agent in the ROI significantly. The differences in molar concentration of the applied GBCAs resulted in different injection volumes affecting contrast agent bolus characteristics. This cannot be easily corrected for; in fact, the shorter bolus geometry may also result in more complex effects.

Just reducing the injection rate of the $1.0 \mathrm{M}$ agent by $50 \%$ or increasing the rate by $100 \%$ for the $0.5 \mathrm{M}$ agents does not adjust the bolus geometry. Especially, increasing injection rate may lead to drawbacks because the achieved signal is not linear to the concentration but actually decreases at high concentrations. Morelli et $\mathrm{al}^{24}$ could show that doubling the injection rate does not lead to a significant increase in SNR and diagnostic window. ${ }^{39}$ For static MRA, $0.07 \mathrm{mmol} / \mathrm{kg}$ are less than the recommended single dose; however, this low-dose approach accounts for all of the compared contrast agents and thus does not constitute a drawback for any of the agents as compared with the others. Our results show that, under the given conditions, the increased Gd concentration of Gadobutrol seems to be of higher importance than does the interaction with blood proteins of Gadobenate dimeglumine for carotid MRA. Recent studies showed that increasing the injection rate and thus the concentration within the blood does not necessarily lead to a higher signal. ${ }^{40}$ The in vitro higher relaxivity of Gadobenate dimeglumine is not reflected in qualitative and quantitative in vivo results. This might be caused by the fact that data acquisition in carotid MRA happens approximately 15 to 20 seconds after contrast agent injection. Morphologic imaging, where Gadobenate dimeglumine shows not significantly different or higher SNR values as compared with Gadobutrol, ${ }^{12,35,41}$ is performed approximately 3 to 5 minutes after contrast agent application. This leads to the assumption that the maximal relaxivity of Gadobenate dimeglumine is not reached directly after injection. In fact, it was already observed with other contrast agents featuring protein binding that the full relaxivity of these agents change over time; that is, relaxivity is lower in the arterial first pass than in the postbolus phase. ${ }^{42}$ This might be related to incomplete contribution of protein binding in the highly concentrated contrast bolus, where the actual albumin excess might not be sufficient for Gadobenate dimeglumine to allow for reaching its maximum relaxivity. ${ }^{43}$ However, this effect can only be assumed for the first minutes after contrast agent application because Neira et $\mathrm{al}^{44}$ could show a decrease in blood R1 within 10 minutes after contrast agent application. The lower in vitro relaxivity of Gadoterate meglumine is consistently reflected by qualitative and quantitative in vivo results. Results of the qualitative 
image quality reading comparing the agents side by side are perfectly supported by the quantitative measurements. The significantly higher SNR and CNR of MRA data sets acquired with Gadobutrol lead to a significantly higher qualitative rating of these data sets. However, CNR values in MRA are of lesser importance because reading these data sets is mostly done after background subtraction. The different ratio in between SNR values for the different agents at different locations can be explained in several ways, for example, the nonlinearity of signal as compared with contrast agent concentration and the variation of signal at different flow velocities. Both are true when comparing measurements at the proximal and distal ICA. Partial volume effects in smaller vessels and the coverage with different coils can explain the differences in SNR at the evaluated levels achieved with all GBCAs. ${ }^{33,45}$

Edge blurring, as it theoretically occurs if only the center parts of $\mathrm{k}$-space are sampled during the presence of contrast agent in the ROI, could be expected because of the small injected volume when using Gadobutrol. Indeed, it was neither quantitatively measurable nor reflected in an impaired qualitative image quality, and no significant differences occurred comparing the 3 evaluated agents. This leads to the conclusion that $0.07 \mathrm{mmol} / \mathrm{kg} \mathrm{BW}$ of a $1.0 \mathrm{M} \mathrm{GBCA}$ injected at $2.0 \mathrm{~mL} / \mathrm{s}$ serves with a sufficiently long contrast agent bolus to avoid disturbing edge blurring; that is, contrast agent is present in the vascular territory of interest during the entire $\mathrm{k}$-space sampling in an adequate amount.

Since the introduction of dynamic MRA, this technique has undergone great improvement in spatial and temporal resolution and, nowadays, can add valuable information to an MRA examination. However, there is still a trade-off between dynamic information and spatial resolution, meaning, that dynamic MRA cannot completely replace static MRA presently. To further push the image quality of dynamic MRA, this technique benefits from dedicated contrast agents as well. ${ }^{46}$ Signal-to-noise ratio evaluation of dynamic MRA data sets again showed higher values achieved with a highly concentrated GBCA as compared with the standard 0.5 M GBCA. The fact that SNR values achieved with dynamic MRA differ from those measured in static MRA and that the ratio between all agents is different from this measured in static MRA is sequence inherent. The technique we used for dynamic MRA relies on view sharing where only the center part of $\mathrm{k}$-space, which encodes for the contrast in the image, is sampled for every point in time, and the peripheral parts of $\mathrm{k}$-space are shared between different frames. In dynamic MRA, the SNR differences between Gadobenate dimeglumine and Gadoterate meglumine as compared with Gadobutrol are less pronounced than in static MRA. This can be rationalized by the used sequence settings and the injection parameters. The sequence used for dynamic MRA facilitates in setting the size of the central region A of k-space, which is sampled in every time frame, and the sampling density of the peripheral part B of $\mathrm{k}$-space. Because we selected a size of $17 \%$ of the entire $\mathrm{k}$-space for region $\mathrm{A}$, which is relatively small, some parts of the central $\mathrm{k}$-space that contribute to the contrasts in the image are sampled as part of the periphery. Sampling density of $20 \%$ for region $\mathrm{B}$ in combination with a temporal resolution of 1.86 seconds per frame ends up in a temporal footprint for sampling the entire k-space data for 1 time frame of approximately 9 seconds. An injection rate of $2 \mathrm{~mL} / \mathrm{s}$ of $0.03 \mathrm{mmol} / \mathrm{kg} \mathrm{BW}$ ( $\sim 4.5 \mathrm{~mL}$ for Gadobenate dimeglumine and Gadoterate meglumine $/ \sim 2.3 \mathrm{~mL}$ for Gadobutrol in a $75 \mathrm{~kg}$ participant) results in a bolus shorter than 1 temporal footprint. For a very short bolus, as it is a fact when using Gadobutrol, it means that not all central $\mathrm{k}$-space sampling is done during the presence of a contrast agent. However, the resulting signal is averaged from the entire temporal footprint, resulting in a lower signal in the final image than the actual reached maximum during acquisition. On the other hand, for a bolus long enough for a footprint, it results in a higher mean signal over the sampling time.

Findings at the later time frames of signal curves beyond the arterial peak enhancement show the effect of interaction of Gadobenate dimeglumine with blood proteins. Signal curves of Gadobutrol and Gadoterate meglumine both flatten with time. The curve of Gadobenate dimeglumine also flattens but much less than that of both comparators, most likely because of the prolonged presence of Gadobenate dimeglumine in the blood pool. At the last sampled time point ( $98 \mathrm{sec}$. p.i.), the signal curves of Gadobutrol and Gadobenate dimeglumine nearly intersect; this might explain the finding of other publications that show no significant differences between Gadobutrol and Gadobenate dimeglumine in peripheral MRA.

A limitation of this study is the exclusive evaluation of volunteer data sets without any pathologic findings. Thus, evaluation of the influence of different SNR on diagnostic accuracy was not possible. However, this was not an aim of the current study and will be evaluated in the future. Three different examinations with required intervals in between in a patient with potentially severe carotid artery disease are delaying necessary treatment and are thus ethically unjustifiable.

\section{CONCLUSION}

This study indicates that, in static and dynamic MRA of the carotid arteries, a contrast agent that features a higher Gd concentration shows higher qualitative image quality and higher SNR and CNR as compared with $0.5 \mathrm{M}$ agents, regardless of whether they feature protein interaction/binding or not. Because we could not confirm the finding that contrast agents with at least temporary binding to blood components are beneficial for morphologic imaging for MRA applications, it seems that the high relaxivity of these agents due to concentration differences of contrast agent molecules and human albumin in the first-pass bolus is reached after some time of interaction with blood components but not directly after injection. However, these agents might be superior because of the higher relaxivity in later phase imaging. Our results reflect findings in carotid MRA, and the evaluated characteristics might be different in other vascular territories that are imaged with an extended delay after injection. Using a highly concentrated GBCA enables comprehensive static and dynamic MRA imaging of the head and neck arteries without the need to exceed the recommended dosage of $0.1 \mathrm{mmol} / \mathrm{kg} \mathrm{BW}$.

\section{ACKNOWLEDGMENTS}

The authors thank Denise Steffinger, Markus Bregler, and Michele Picciolo for their continuous tremendous help during the execution of all MRI examinations, Martin Rohrer for his input on molecular principles of contrast agents, and Gerhard Laub for his input on MR sequence basics.

\section{REFERENCES}

1. Prabhakaran P, Ajay VS, Prabhakaran D, et al. Global cardiovascular disease research survey. J Am Coll Cardiol. 2007;50:2322-2328.

2. Reddy KS. Cardiovascular disease in non-Western countries. $N$ Engl J Med. 2004;350:2438-2440.

3. Reddy KS, Yusuf S. Emerging epidemic of cardiovascular disease in developing countries. Circulation. 1998;97:596-601.

4. Remonda L, Senn P, Barth A, et al. Contrast-enhanced 3D MR angiography of the carotid artery: comparison with conventional digital subtraction angiography. AJNR Am J Neuroradiol. 2002;23:213-219.

5. Sundgren PC, Sunden P, Lindgren A, et al. Carotid artery stenosis: contrastenhanced MR angiography with two different scan times compared with digital subtraction angiography. Neuroradiology. 2002;44:592-599.

6. Anzalone N, Scomazzoni F, Castellano R, et al. Carotid artery stenosis: intraindividual correlations of 3D time-of-flight MR angiography, contrastenhanced MR angiography, conventional DSA, and rotational angiography for detection and grading. Radiology. 2005;236:204-213.

7. Nael K, Ruehm SG, Michaely HJ, et al. High spatial-resolution CE-MRA of the carotid circulation with parallel imaging: comparison of image quality between 2 different acceleration factors at 3.0 tesla. Invest Radiol. 2006;41:391-399.

8. Kramer H, Runge VM, Morelli JN, et al. Magnetic resonance angiography of the carotid arteries: comparison of unenhanced and contrast enhanced techniques. Eur Radiol. 2011;21:1667-1676. 
9. Lohan DG, Tomasian A, Saleh RS, et al. Ultra-low-dose, time-resolved contrastenhanced magnetic resonance angiography of the carotid arteries at 3.0 tesla. Invest Radiol. 2009;44:207-217.

10. McCarthy RM, Deshpande VS, Beohar N, et al. Three-dimensional breathhold magnetization-prepared TrueFISP: a pilot study for magnetic resonance imaging of the coronary artery disease. Invest Radiol. 2007;42:665-670.

11. Achenbach M, Figiel JH, Burbelko M, et al. Prospective comparison of image quality and diagnostic accuracy of 0.5 molar gadobenate dimeglumine and 1.0 molar gadobutrol in contrast-enhanced run-off magnetic resonance angiography of the lower extremities. J Magn Reson Imaging. 2010;32:1166-1171.

12. Essig M, Lodemann KP, Le-Huu M, et al. Intraindividual comparison of gadobenate dimeglumine and gadobutrol for cerebral magnetic resonance perfusion imaging at 1.5 T. Invest Radiol. 2006;41:256-263.

13. Nikolaou K, Kramer H, Grosse C, et al. High-spatial-resolution multistation MR angiography with parallel imaging and blood pool contrast agent: initial experience. Radiology. 2006;241:861-872.

14. Rohrer M, Bauer H, Mintorovitch J, et al. Comparison of magnetic properties of MRI contrast media solutions at different magnetic field strengths. Invest Radiol. 2005;40:715-724.

15. Pintaske J, Martirosian P, Graf $\mathrm{H}$, et al. Relaxivity of gadopentetate dimeglumine (Magnevist), gadobutrol (Gadovist), and gadobenate dimeglumine (MultiHance) in human blood plasma at 0.2, 1.5, and 3 tesla. Invest Radiol. 2006;41:213-221.

16. Kramer H, Runge VM, Naul LG, et al. Brain MRI with single-dose $(0.1 \mathrm{mmol} / \mathrm{kg})$ gadobutrol at $1.5 \mathrm{~T}$ and $3 \mathrm{~T}$ : comparison with $0.15 \mathrm{mmol} / \mathrm{kg}$ gadoterate meglumine. AJR Am J Roentgenol. 2010;194:1337-1342.

17. Cowper S. Nephrogenic Fibrosing Dermopathy [NFD/NSF Web site]. 2001-2009: Available at http://www.icnfdr.org. Accessed August 10, 2012.

18. Thomsen HS. Nephrogenic systemic fibrosis: a serious late adverse reaction to gadodiamide. Eur Radiol. 2006;16:2619-2621.

19. Katoh M, Spuentrup E, Stuber M, et al. Flow targeted 3D steady-state freeprecession coronary MR angiography: comparison of three different imaging approaches. Invest Radiol. 2009;44:757-762.

20. Herborn CU, Runge VM, Watkins DM, et al. MR angiography of the renal arteries: intraindividual comparison of double-dose contrast enhancement at 1.5 T with standard dose at 3 T. AJR Am J Roentgenol. 2008;190:173-177.

21. Jourdan C, Heverhagen JT, Knopp MV. Dose comparison of single- vs. doubledose in contrast-enhanced magnetic resonance angiography of the carotid arteries: intraindividual cross-over blinded trial using Gd-DTPA. J Magn Reson Imaging. 2007; 25:557-563.

22. Thurnher SA, Capelastegui A, Del Olmo FH, et al. Safety and effectiveness of single- versus triple-dose gadodiamide injection- enhanced MR angiography of the abdomen: a phase III double-blind multicenter study. Radiology. 2001 219:137-146.

23. Finn JP, Baskaran V, Carr JC, et al. Thorax: low-dose contrast-enhanced threedimensional MR angiography with subsecond temporal resolution-initial results. Radiology. 2002;224:896-904.

24. Morelli JN, Runge VM, Ai F, et al. Magnetic resonance evaluation of renal artery stenosis in a swine model: performance of low-dose gadobutrol versus gadoterate meglumine in comparison with digital subtraction intra-arterial catheter angiography. Invest Radiol. 2012;47:376-382.

25. Wuesten O, Morelli JN, Miller MW, et al. MR angiography of carotid artery aneurysms in a porcine model at 3 tesla: comparison of two different macrocyclic gadolinium chelates and of dynamic and conventional techniques. J Magn Reson Imaging. 2012;36:1203-1212.

26. Hadizadeh DR, Kukuk GM, Fahlenkamp UL, et al. Simultaneous MR arteriography and venography with blood pool contrast agent detects deep venous thrombosis in suspected arterial disease. AJR Am J Roentgenol. 2012;198: 1188-1195.

27. Kramer U, Fenchel M, Laub G, et al. Low-dose, time-resolved, contrastenhanced 3D MR angiography in the assessment of the abdominal aorta and its major branches at 3 tesla. Acad Radiol. 2010;17:564-576.

28. Dietrich O, Raya JG, Reeder SB, et al. Measurement of signal-to-noise ratios in MR images: influence of multichannel coils, parallel imaging, and reconstruction filters. J Magn Reson Imaging. 2007;26:375-385.
29. Anzidei M, Napoli A, Marincola BC, et al. High-resolution steady state magnetic resonance angiography of the carotid arteries: are intravascular agents necessary?: feasibility and preliminary experience with gadobenate dimeglumine. Invest Radiol. 2009;44:784-792.

30. Bueltmann E, Erb G, Kirchin MA, et al. Intra-individual crossover comparison of gadobenate dimeglumine and gadopentetate dimeglumine for contrastenhanced magnetic resonance angiography of the supraaortic vessels at 3 tesla. Invest Radiol. 2008;43:695-702.

31. Nael K, Fenchel M, Krishnam M, et al. 3.0 tesla high spatial resolution contrast-enhanced magnetic resonance angiography (CE-MRA) of the pulmonary circulation: initial experience with a 32-channel phased array coil using a high relaxivity contrast agent. Invest Radiol. 2007;42:392-398.

32. Berg F, Bangard C, Bovenschulte H, et al. Feasibility of peripheral contrastenhanced magnetic resonance angiography at 3.0 tesla with a hybrid technique: comparison with digital subtraction angiography. Invest Radiol. 2008;43: 642-649.

33. Kramer H, Michaely HJ, Matschl V, et al High-resolution magnetic resonance angiography of the lower extremities with a dedicated 36-element matrix coil at 3 tesla. Invest Radiol. 2007;42:477-483.

34. Kramer H, Zenge M, Schmitt P, et al. Peripheral magnetic resonance angiography (MRA) with continuous table movement at $3.0 \mathrm{~T}$ : initial experience compared with step-by-step MRA. Invest Radiol. 2008;43:627-634.

35. Anzalone N. Comparative studies of different gadolinium agents in brain tumors: differences between gadolinium chelates and their possible influence on imaging features. AJNR Am J Neuroradiol. 2010;31:981-982.

36. Anzalone N, Gerevini S, Scotti R, et al. Detection of cerebral metastases on magnetic resonance imaging: intraindividual comparison of gadobutrol with gadopentetate dimeglumine. Acta Radiol. 2009;50:933-940.

37. Le Duc G, Corde S, Charvet AM, et al. In vivo measurement of gadolinium concentration in a rat glioma model by monochromatic quantitative computed tomography: comparison between gadopentetate dimeglumine and gadobutrol. Invest Radiol. 2004;39:385-393.

38. Huppertz A, Rohrer M. Gadobutrol, a highly concentrated MR-imaging contrast agent: its physicochemical characteristics and the basis for its use in contrast-enhanced MR angiography and perfusion imaging. Eur Radiol. 2004; 14(suppl 5):M12-18.

39. Husarik DB, Bashir MR, Weber PW, et al. Contrast-enhanced magnetic resonance angiography: first-pass arterial enhancement as a function of gadoliniumchelate concentration, and the saline chaser volume and injection rate. Invest Radiol. 2012;47:121-127.

40. Wilson GJ, Bastawrous S, Bhargava P, et al. Relaxivities of Protein and nonProtein Binding Gd Agents at High Concentrations in Blood: Relaxation Measurements. Utrecht, the Netherlands: MRA Club; 2012. Abstract.

41. Seidl Z, Vymazal J, Mechl M, et al. Does higher gadolinium concentration play a role in the morphologic assessment of brain tumors? Results of a multicenter intraindividual crossover comparison of gadobutrol versus gadobenate dimeglumine (the MERIT Study). AJNR Am J Neuroradiol. 2012;33:1050-1058.

42. Port $\mathrm{M}$, Corot $\mathrm{C}$, Violas X, et al. How to compare the efficiency of albuminbound and nonalbumin-bound contrast agents in vivo: the concept of dynamic relaxivity. Invest Radiol. 2005;40:565-573.

43. Rohrer M. MRI contrast media-introduction and basic properties of the blood pool agent vasovist. In: Leiner TG, Rohrer M, Schienberg SO, eds. Clinical Blood Pool MR Imaging. Heidelberg, Germany: Springer Medizinverlag; 2008:3-15.

44. Neira C, Anzidei M, Napoli A, et al. Investigation of the longitudinal relaxation rate of blood after gadobenate dimeglumine administration: sequence optimization, dynamic acquisition, and clinical impact for contrast-enhanced MR angiography of the carotid arteries. Invest Radiol. 2011;46:774-782.

45. Schultz E, Felix R. Phantom measurements of spatial resolution and the partialvolume-effect in computer tomography (author's transl) [in German]. Rofo. 1978; 129:673-678.

46. Kramer H, Michaely HJ, Requardt M, et al. Effects of injection rate and dose on image quality in time-resolved magnetic resonance angiography (MRA) by using 1.0 M contrast agents. Eur Radiol. 2007;17:1394-1402. 